\section{MS12-04 Making the most out of screening experiments}

Jia Tsing $\mathrm{Ng}^{1}$, Carien Dekker ${ }^{2}$, Markus Kroemer ${ }^{2}$, Michael Osborne $^{3}$, Frank von Delft ${ }^{1,4,5}$

1. Structural Genomics Consortium, University of Oxford, Roosevelt Drive, Oxford OX3 7DQ, England

2. Novartis Institute for Biomedical Research, Novartis Campus, Postfach, CH-4056 Basel, Switzerland

3. Department of Engineering Science, University of Oxford, Parks Road, Oxford OX1 3PJ, England

4. Diamond Light Source Ltd, Harwell Science and Innovation Campus, Didcot OX11 0QX, England

5. Department of Biochemistry, University of Johannesburg, Auckland Park 2006, South Africa

email: jiatsing.ng@dtc.ox.ac.uk

We present computational tools that extract information from standard sparse-matrix screens, to help experimenters make informed decisions in subsequent crystallization effort, even if there were no crystals in the initial screen. Our method is based on the objective description of crystallization droplets using textons in $\mathrm{Ng}$ et al. (2014).

Firstly, we aid in the identification of crystals or microcrystals by ranking droplets according to their likelihood of containing crystalline behaviour. This allows users to view droplets in a more meaningful order and prioritise attention and time to what is likely to matter most.

Secondly, we describe screening experiments by the collective precipitation patterns across the screen. This enables us to cluster historical experiments at the Structural Genomics Consortium, Oxford by their precipitation behaviour. Each cluster has a different distribution of crystallization conditions that gave hits, which is then used to identify conditions for optimization for a new protein that falls into the same cluster.

Thirdly, based on the idea of Collins et al. (2005), we automatically generate an analysis of conditions that produced clear drops. These conditions can be used to design an alternative protein formulation buffer for further stabilization of the sample. A stable formulation allows for crystallization experiments at higher protein concentration. We present cases for each of these applications and how they have enabled the crystallization of difficult targets, and/or improved the crystal quality. A centralised software, TeXRank, presents the above analysis of screening data and allows users to view and annotate their images.

References:

Collins, B., Stevens, R. C., \& Page, R. (2005). Acta Crystallogr. Sect. F Struct. Biol. Cryst. Commun. 61, 1035-1038.

Ng, J. T., Dekker, C., Kroemer, M., Osborne, M., \& von Delft, F. (2014). Acta Crystallogr. Sect. D Biol. Crystallogr. 70, 2702-2718.

Keywords: screening, machine vision, automated analysis
MS12-05 Protein crystal nucleation induced by ionic liquid-functionalized mineral particles

Magdalena Kowacz ${ }^{1}$, Mateusz Marchel ${ }^{1}$, Lina Juknaite ${ }^{2}$, Ana Luísa Carvalho ${ }^{2}$, Maria João Romão ${ }^{2}$, José M. S. S. Esperança ${ }^{1}$, Luís Paulo N. Rebelo ${ }^{1}$

1. Instituto de Tecnologia Química e Biológica - António Xavier, Universidade Nova de Lisboa, Av. da República, 2780-157 Oeiras, Portugal.

2. UCIBIO, REQUIMTE, Departamento de Química, Faculdade de Ciências e Tecnologia, Universidade Nova de Lisboa, 2829-516 Caparica, Portugal

email: magda@itqb.unl.pt

Nucleation is a critical step that determines the outcome of the entire crystallization process. Therefore, finding an effective nucleant for protein crystallization is of utmost importance for structural biology that relies on good quality crystals to solve the 3D structures of macromolecules. We show that crystalline barium sulfate $\left(\mathrm{BaSO}_{4}\right)$ with etched and/or ionic liquid (IL) functionalized surface can: (1) induce protein nucleation at protein concentrations well below the concentration needed to promote crystal growth at control conditions, (2) can shorten nucleation time, (3) increase growth rate and finally (4) can improve protein crystal morphology. These effects are shown for Hemoglobin, Myoglobin, Trypsin, Proteinase K, RNase A and Lysozyme.

Surface characteristics of our effective heterogeneous nucleant - porosity (dissolution pits), disorder (partially broken crystal contacts and liquid-like IL coating) and solid strain - support previously proposed surface-supported mechanisms of nucleation. These refer to protein entrapment and induction of supersaturation spikes inside of the pores, providing surface-protein binding sites at a right distance to match protein-protein spacing in the crystal or supporting of entropy-driven nucleation induced by strained mismatched solid support.

Moreover this work shows for the first time the effect of water-immiscible ionic liquids on crystallization of biomacromolecules from aqueous solution. This is enabled by the use of the hydrophobic ILs immobilized at the surface of a hydrophilic mineral. Our results indicate that partitioning of ionic liquid and of protein amphiphiles to solid surface is governed by the same mechanisms. Therefore ILs can serve as simple models giving some insight into protein-solid interactions. Both, ILs and biomacromolecules interact with the hydrophilic solid through their polar groups, while hydrophobic compartments determine their tendency to separate from aqueous solution and drive further hydrophobic association near the solid surface. ILs tune protein partitioning to the solid support by providing matching H-bonding sites as well as non-polar patches at hydrophilic surface.

Modification of the solid surface nanotopography and its functionalization are not restricted to the particular mineral-ILs system, so that their impact on protein conformational changes can be further explored.

The authors acknowledge financial support from Fundação para a Ciência e a Tecnologia (FCT). 


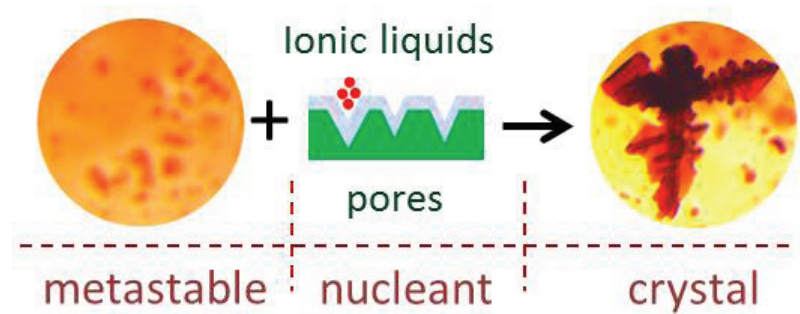

Figure 1. Mineral particles with porous surface and ionic liquid coating induce protein crystallization in a metastable zone.

Keywords: solid-supported crystallization, crystallization from metastable zone, heterogeneous nucleant
MS13. New instrumentation,

methods and approaches in

inorganic crystallography

Chairs: Anton Meden, Damien Jacob
MS13-01 Complex metal hydrides: When powder diffraction needs help

Radovan Černý ${ }^{1}$, Pascal Schouwink ${ }^{1}$, Yolanda Sadikin ${ }^{1}$, Matteo Brighi $^{1}$, Emilie Didelot ${ }^{1}$

1. Laboratory of Crystallography, DQMP, University of Geneva, 24 Quai Ernest-Ansermet, CH-1211, Geneva Switzerland

email: Radovan.Cerny@unige.ch

'Real life' energy-related materials such as solid-state hydrogen storage compounds or components of electrochemical cells are usually polycrystalline, poorly crystallized, highly reactive and dynamic systems. Powder diffraction at modern high brilliance X-ray sources is the most useful tool to investigate such systems because it is easy, fast and extremely versatile with respect to measurement conditions as well as in situ setups. However, powder diffraction applied to these systems rapidly reaches its limits due to the bad crystallinity of samples prepared by mechanochemistry as well as due to the method itself. We will show how a complementary approach combining powder diffraction with non-diffraction methods such as vibrational spectroscopy, thermal analysis and supported by ab initio solid state calculations allows overcoming these limitations ${ }^{1}$.

The investigation of samples containing several novel crystalline phases of uncertain chemical composition appears as a problem with no unique solution. We will show that a purposeful use of various samples of different nominal compositions, powder diffraction patterns measured while thermally decomposing each phase (T-ramping) ${ }^{2}$, direct space methods for structure solution and refinement by $\mathrm{ab}$ initio solid state calculations does provide detailed structural information of novel compounds even for hydrogen atoms ${ }^{3}$.

The recently developed possibility of performing mechanochemistry, usually a "black box" approach, on reactive samples under the beam will also be discussed shortly. Ball milling mapped by in situ powder diffraction provides a means of "watching" the synthesis of novel materials, and it is the latest tool we have applied to follow the reactions of complex hydrides inside the reaction jars, capturing intermediate phases and tuning the milling conditions to selectively favour phase yields ${ }^{3}$.

\section{References}

1 Schouwink P. and Cerny R. Complex hydrides: When powder diffraction needs help. Chimia, 68 (2014) nr. 1/2, 38-44, dx.doi.org/10.2533/chimia.2014.38 